\title{
Brongniartia balsensis (Leguminosae, Papilionoideae), una Especie Nueva de la Depresión del Río Balsas, México
}

\author{
Jaime Jiménez Ramírez \\ Departamento de Biología Comparada, Facultad de Ciencias, Universidad Nacional Autónoma \\ de México, Apartado Postal 70-399, Delegación Coyoacán 04510, México, D.F., México \\ jjr@hp.feiencias.unam.mx
}

Karla Vega Flores

Herbario y Jardín Botánico, Benemérita Universidad Autónoma de Puebla, Edificio 76, Ciudad Universitaria, Blvd. Valsequillo y Av. San Claudio s/n, Col. San Manuel, Puebla,

72590, Puebla, México

\section{Ramiro Cruz Durán}

Departamento de Biología Comparada, Facultad de Ciencias, Universidad Nacional Autónoma de México, Apartado Postal 70-399, Delegación Coyoacán 04510, México, D.F., México

Resumen. Se describe e ilustra a Brongniartia balsensis J. Jiménez Ramírez \& K. Vega (Leguminosae, Papilionoideae), una especie nueva y endémica de la Depresión del Balsas. El nuevo taxón es similar a B. guerrerensis J. Jiménez Ramírez \& J. L. Contreras, pero difiere de esta especie por tener un mayor número de folíolos y por presentar pedicelos, bractéolas, cáliz, estandarte, alas y quilla de mayor tamaño, además de tener diferencias en la forma del estandarte, el color de la quilla (verde) y poseer una legumbre ligeramente alada.

Abstract. Brongniartia balsensis J. Jiménez Ramírez \& K. Vega (Leguminosae, Papilionoideae), a new and endemic species from the Balsas Depression, is described and illustrated. This species is similar to B. guerrerensis J. Jiménez Ramírez \& J. L. Contreras, but differs from it by having a greater number of leaflets and larger pedicels, bracteoles, calyx, standard, wings, and keel; there are also differences in the form of the standard, the color of the keel (green), and the possession of a lightly winged legume.

Key words: Balsas Depression, Brongniartia, IUCN Red List, Leguminosae, Mexico, Papilionoideae.

El género Brongniartia Kunth con distribución desde Texas, México y Centroamérica hasta los Andes (McVaugh, 1987) incluye alrededor de 65 especies (Dorado, 1999; Dorado \& Arias, 2006), casi todas endémicas de México.

Rydberg (1923) ubica a Brongniartia en la subtribu Brongniartianae, en tanto McVaugh (1987) la incluye en la tribu Brongniartieae. El género está constituido por árboles o arbustos con hojas imparipinnadas, estípulas pronto caedizas, flores generalmente axilares, solitarias o en grupos de dos a siete, o en racimos terminales o panículas, cáliz brevemente bilabiado con bractéolas, estandarte orbicular u obovado, el estambre superior libre, estilo filiforme, incurvado, fruto aplanado, en ocasiones con margen alado en la parte vexilar (McVaugh, 1987).

En el "Estudio florístico de la porción guerrerense de la cuenca del río Balsas", que se desarrolla en el Herbario de la Facultad de Ciencias (FCME), Universidad Nacional Autónoma de México (UNAM), se detectó una especie del género Brongniartia afín al grupo Sericeae de Rydberg (1923). Esta especie tiene parecido morfológico con $B$. guerrerensis J. Jiménez Ramírez \& J. L. Contreras, que fue ubicada por Jiménez y Contreras (1989) en el grupo Sericeae de Rydberg (1923).

Brongniartia balsensis J. Jiménez Ramírez \& K. Vega, sp. nov. TIPO: México. Guerrero: Mpio. Copalillo, $1 \mathrm{~km}$ antes de intersección entre el poblado y balneario de Papalutla, 30 abr. 2007, J. Jiménez 1668 (holotipo, FCME; isotipos, MEXU, MO). Figura 1.

Haec species Brongniartiae guerrerensi J. Jiménez Ramírez \& J. L. Contreras affinis, a qua imprimis cortice brunneo rubrove, stipulis oblongis, pedicello et calyce majoribus, vexillo orbiculari majore brunneo, carina viridi majore et legumine alato differt.

Arbustos (1-)3-3.5(-4.5) m de alto, generalmente con 3-12 ejes principales o árboles hasta $4 \mathrm{~m}$; corteza 


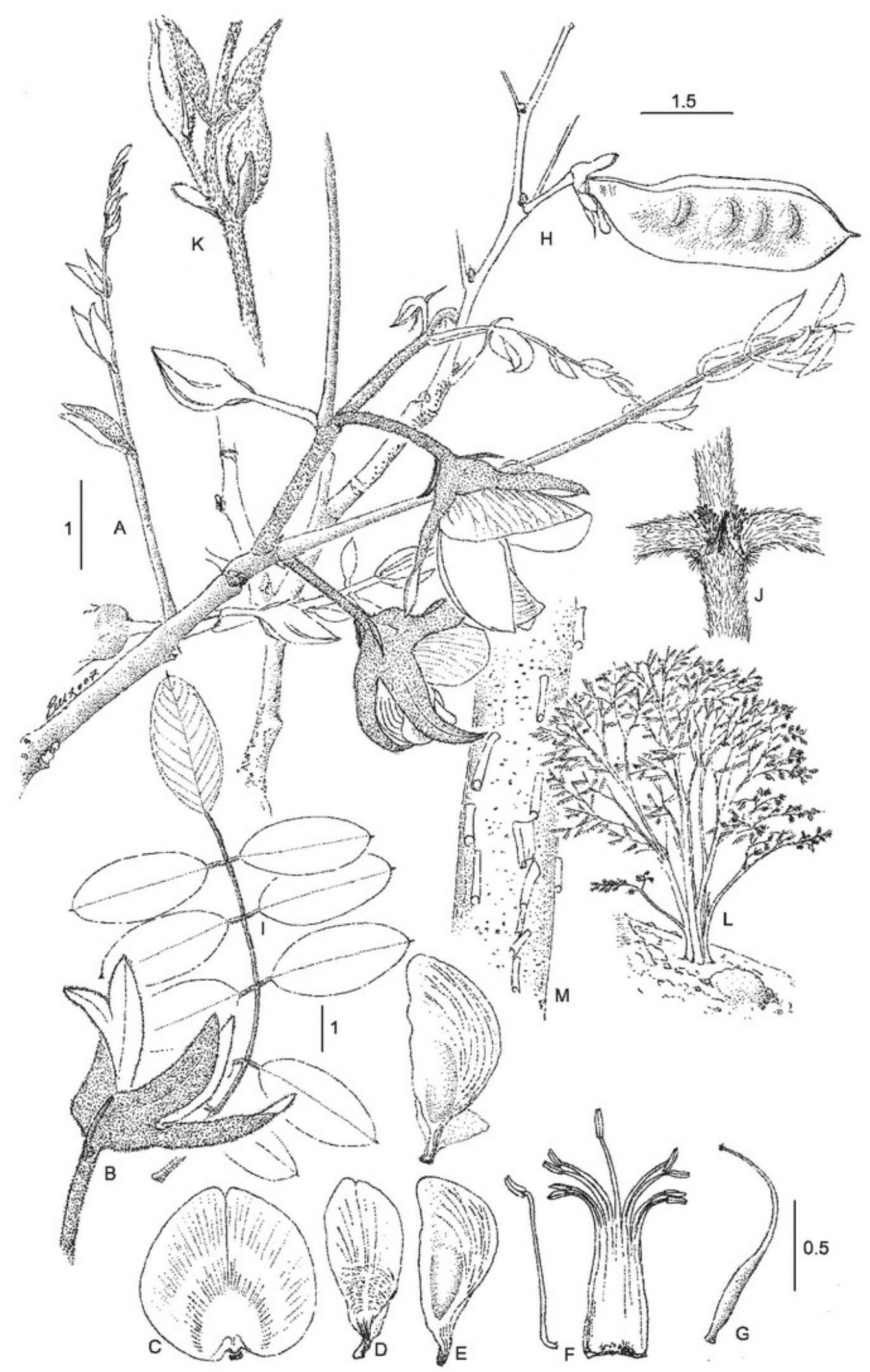

Figura 1. Brongniartia balsensis J. Jiménez Ramírez \& K. Vega. —A. Rama con flores. —B. Cáliz y bractéolas. —C. Estandarte. -D. Ala. -E. Pétalo de la quilla. -F. Androceo. - G. Gineceo. - H. Fruto. -I. Folíolos maduros. -J. Estipelas. -K. Estípulas. - L. Hábito. - M. Corteza. (A-G y K, Jiménez 1668, FCME; H, Vega 1105, FCME; I, J, Cruz et al. 7033, FCME). Todas las escalas en centímetros. 
café a rojiza con abundantes lenticelas blancas, exfoliante en grandes fragmentos (en individuos jóvenes la corteza es blanca y corchosa, generalmente en la parte basal del tallo); ramillas blanco-seríceas. Hojas (9-)12.5-16(-19) cm, imparipinnadas, con 11 a 15(17) folíolos; estípulas ca. $5 \times 1.5 \mathrm{~mm}$, oblongas, envés blanco-seríceo, pronto caedizas; pecíolos (1.3-) 1.5-3.3(-5) cm, blanco-seríceos; raquis seríceo; folíolos 2.4-3.5(-4) $\times$ 1.1-2(-2.3) em, elípticos, pubescentes, blanco-seríceos cuando jóvenes, al madurar verdes en el haz, ápice con mucrón de hasta $1 \mathrm{~mm}$, base obtusa, peciólulos 3-4 mm, blancoseríceos; estipelas hasta $1 \mathrm{~mm}$, seríceas en el envés, anaranjadas. Flores axilares, 2 por axila, opuestas (generalmente se fecunda una); pedicelos (1-)1.41.9(-2.6) cm, blanco-seríceos; cáliz 19.1-20.9 mm, blanco-seríceo, tubo 6.7-7.3 mm; bractéolas (2.5-)4$8.2 \mathrm{~mm}$, filiformes, blanco-seríceas; corola marrón; estandarte marrón con mácula verde, 19.2-19.6 × 18.5-19.5 mm, orbicular, ápice emarginado con uña 3.4-3.7 mm, ala (21.5-)22.1 × (8.2-)9.1-11 mm con uña $3.5-4.4 \mathrm{~mm}$, quilla verde, $21.8-23.2 \times 9.4-11.1$ $\mathrm{mm}$, parte unida hasta $1 / 4$ de su longitud, ca. $7.5 \mathrm{~mm}$, uña $3.2-3.4 \mathrm{~mm}$, presenta una aurícula en la base 2$3.1 \mathrm{~mm}$, androceo ca. $22 \mathrm{~mm}$, parte unida $15.5-17.4$ $\mathrm{mm}$; pistilo 26.1-26.7 mm, glabro, ovario 12-12.6 $\mathrm{mm}$, ́vvulos 4 a 6, estilo 14-15.8 mm. Legumbre (5.5-) 6-6.7 $\times 1.8-2.2 \mathrm{~cm}$, aplanada, sutura carinal en forma de ala de hasta $2 \mathrm{~mm}$ de ancho; semillas 4 a 5 por fruto.

Distribución. Brongniartia balsensis restringe su distribución a la porción oriental de la Depresión del Balsas en el estado de Guerrero, en altitudes de (699-) 762-809 m y solamente se conocen dos poblaciones, una en Papalutla de unos 100 individuos y otra en las cercanías de Tlalcozotitlán con unos 40 individuos, por ello es una especie en peligro crítico (Critically Endangered o CR), según los criterios de la UICN (2001).

Hábitat y fenología. Esta especie se presenta en bosque tropical caducifolio en laderas con substratos kársticos. Se le encuentra asociada a Pseudosmodingium perniciosum (Kunth) Engler, Bursera Jacquin ex L., Plumeria rubra L., Neobuxbaumia Backeberg, Mimosa L., Croton L., Ceiba parvifolia Rose, Manihot Miller, Opuntia Miller, Cnidoscolus Pohl, Senna wislizeni (A. Gray) H. S. Irwin \& Barneby, Dalembertia populifolia Baillon, Stemmadenia pubescens Bentham, Lonchocarpus Kunth, Jatropha elbae J. Jiménez Ramírez, Randia L., Acacia coulteri Bentham y Nissolia leiogyne Sandwith. La floración se presenta desde finales de abril hasta mediados de mayo (aunque en un individuo, se formaron flores en fechas posteriores en algunas ramas donde se cortaron previamente, en el mes de abril, las ramillas para herborizar), mientras las hojas inician su desarrollo, en tanto que la fructificación ocurre de mayo a julio.

Etimología. El epíteto específico hace alusión a la depresión del Balsas, una zona rica en endemismos, en particular en la porción que incluye el estado de Guerrero.

Discusión. Las flores axilares, la pubescencia blanco-serícea abundante en las ramillas, los folíolos y el cáliz, los 11 a 15(17) folíolos elípticos y los frutos glabros y alados del nuevo taxón, conducen a ubicarlo en el grupo Sericeae de Rydberg (1923) que incluye a Brongniartia argentea Rydberg, $B$. benthamiana Hemsley, B. lupinoides (Kunth) Taubert, B. magnibracteata Schlechtendal, B. mollis Kunth, B. pringlei Rydberg, B. sericea Schlechtendal y a B. vicioides $\mathrm{M}$. Martens \& Galeotti, posteriormente Jiménez y Contreras (1989) agregan a B. guerrerensis. Sin embargo, McVaugh (1987) señala que existen problemas para delimitar a casi todas las especies del grupo, a excepción de $B$. lupinoides, aunque no menciona nada alrededor de B. magnibracteata y B. mollis, mientras que Calderón y Rzedowski (2001) apuntan que posiblemente $B$. benthamiana y $B$. mollis sean sinónimos de $B$. intermedia Moricand. A pesar de los problemas señalados, la especie nueva presenta una morfología muy diferente a las especies incluidas por Rydberg (1923) en el grupo Sericeae, pues ninguna de ellas, tiene corteza exfoliante como la que se presenta en muchas especies del género Bursera, asimismo carecen de hábito arbóreo en su madurez, de corcho en la base del tallo de las plántulas, de estípulas oblongas, de bractéolas filiformes y de flores color marrón. Aunque, es importante aclarar que $B$. guerrerensis fue incluida recientemente en el grupo Sericeae de Rydberg (1923), tiene corteza exfoliante y habito arbóreo, por lo que será examinada cuidadosamente en los párrafos siguientes.

En el género, solamente se han reportado seis especies arbóreas, Brongniartia glabrata Hooker \& Arnold, B. goldmanii Rose, B. guerrerensis, B. guiengolensis Dorado \& L. Torres, B. montalvoana Dorado \& D. M. Arias y B. parry $i$ Hemsley, de modo que $B$. balsensis es la séptima. Entre ellas, $B$. guerrerensis que tiene corteza exfoliante y corcho en sus ramas, fue ubicada en el grupo Sericeae de Rydberg (1923) por Jiménez y Contreras (1989), por presentar flores axilares, legumbre glabra, ramas, ramillas, folíolos (oblongo-ovados a elípticos) y cálices seríceos, por lo que su comparación con la especie nueva es ineludible, sobre todo porque sus folíolos son similares en el tamaño y forma, así como la de sus pecíolos y peciólulos. Sin embargo, B. guerrerensis 
tiene corteza anaranjada que exfolia en tiras como en Bursera schlechtendalii Engler, estípulas lanceoladas de $2-4 \mathrm{~mm}$, pecíolo de $(0.8-) 1.8-2.5(-3.1) \mathrm{cm}$, folíolos 5 a 11(13), (0.9-)2.3-3(-4.5) × (0.5-)1-2 (-3.6) $\mathrm{cm}$, ovados, oblongo-ovados a elípticos, pedicelos $0.8-1.2 \mathrm{~cm}$, bractéolas lanceoladas de $3-5 \mathrm{~mm}$, cáliz de 1-1.5 cm, corola púrpura, estandarte ovado de ca. $15 \mathrm{~mm}$, alas $10-11 \mathrm{~mm}$, quilla púrpura de 11$13 \mathrm{~mm}$, legumbre 6-8 × 2.5-3 cm, sin ala, en cambio, $B$. balsensis tiene corteza pardo-rojiza que exfolia en placas como en B. papyracea Dorado \& D. M. Arias, estípulas oblongas de $5 \mathrm{~mm}$, pecíolo (1.3-) 1.5-3.3(-5) $\mathrm{cm}$, folíolos 11 a $15(17), 2.4-3.5(-4) \times 1.1-2(-2.3)$ $\mathrm{cm}$, elípticos, pedicelos (1-)1.4-1.9(-2.6) $\mathrm{cm}$, bractéolas filiformes de (2.5-)4-8.2 mm, cáliz 19.1-20.9 mm, corola marrón, estandarte orbicular de 19.2-19.6 $\mathrm{mm}$, alas (21.5-) $22.1 \mathrm{~mm}$, quilla verde de 21.8-23.2 mm y legumbre (5.5-)6-6.7 × 1.8-2.2 cm, con un ala. Es evidente que difieren en el color de la corteza y en el número de folíolos (a pesar de un pequeño traslape), en el tamaño de pedicelos, bractéolas, cáliz, estandarte, alas y quilla, sin olvidar las diferencias en la forma del estandarte, en el color de la quilla y en la legumbre (una especie con ala y otra carente de ella). Además, el período de floración de ambas especies es diferente, B. guerrerensis tiene flores en julio mientras que $B$. balsensis las presenta a finales de abril y en la primera mitad de mayo. El punto no deja de ser importante porque ambas especies se han hallado en localidades cercanas (Tlalcozotitlán, Guerrero), sin embargo, la especie nueva solamente se halla en calizas rosadas y la otra especie parece no tener una afinidad tan específica a un sustrato determinado.

Brongniartia papyracea es la tercera especie del género que presenta tallos exfoliantes, pero se trata de un arbusto de 1-4 m de alto, con corcho en las ramas, que pertenece al grupo Podalyrioides de Rydberg (1923) de acuerdo con Dorado y Arias (2006), por tener estípulas hemicordadas o cordadas y bractéolas lineares y diminutas, por lo que aparentemente no tiene relación cercana con la especie nueva.
Paratipos. MEXICO. Guerrero: Mpio. Copalillo, $1 \mathrm{~km}$ antes de intersección entre el poblado y balneario de Papalutla, J. Jiménez 1669, 1670, 1671, 1672, 1673, 1674 (FCME), K. Vega 1104, 1105, 1106, 1114, 1115, 1116, 1117, $1138,1139,1140$ (FCME); Papalutla, $1.8 \mathrm{~km}$ al NO, R. Cruz, C. Fuentes, L. Céspedes y O. Sánchez 6889, 6891, 6898 (FCME); Atcingo, $1 \mathrm{~km}$ al SE, R. Cruz, C. Fuentes, L. Céspedes y O. Sánchez 7033, 7035 (FCME).

Agradecimientos. A Susana Valencia Ávalos por sus acertadas sugerencias y observaciones hechas al manuscrito, a Martha Martínez Gordillo y Roy Gereau la diagnosis en latín y a José Antonio Hernández del Laboratorio de Microcine, Facultad de Ciencias, UNAM, la digitalización de la figura. Así como a la Dirección General de Asuntos del Personal Académico de la UNAM, por el apoyo económico a través del proyecto IN218206-3 (Programa de Apoyo a Proyectos de Investigación e Innovación Tecnológica [PAPIIT]).

\section{Literatura Citada}

Calderón, G. \& J. Rzedowski. 2001. Flora Fanerogámica del Valle de México. Instituto de Ecología, A. C., Centro Regional de Bajío, Comisión Nacional para el Conocimiento y Uso de la Biodiversidad, Pátzcuaro, Michoacán, México.

Dorado, O. 1999. Brongniartia riesebergii (Fabaceae: Faboideae): A new species from the state of Durango, México. Brittonia 51(4): 398-402.

— \& D. Arias. 2006. Brongniartia papyracea (Fabaceae: Faboideae): A new species from the tropical deciduous forest of southern Jalisco and southwestern Michoacan, Mexico. Brittonia 58(4): 357-361.

Jiménez, J. \& J. L. Contreras. 1989. Una especie nueva de Brongniartia (Leguminosae: Faboideae) de Guerrero, México. Anales Inst. Biol. Univ. Nac. México, Bot. 58: 63-68.

McVaugh, R. 1987. Brongniartia H.B.K. Pp. 297-321 in W. R. Anderson (editor), Flora Novo-Galiciana, Vol. 5. University of Michigan Press, Ann Arbor.

Rydberg, P. A. 1923. Subtribe Brongniartianae. Pp. 186-197 in North American Flora, Vol. 24, Part 3. The New York Botanical Garden, Bronx.

UICN [IUCN]. 2001. IUCN Red List Categories and Criteria, Version 3.1 Prepared by the IUCN Species Survival Commission. IUCN, Gland, Switzerland, and Cambridge, United Kingdom. 


\section{$2 \mathrm{BHL}$ Biodiversity Heritage Library}

Ramírez, Jaime Jiménez, Vega-Flores, Karla, and Cruz Durán, Ramiro. 2009. "Brongniartia balsensis (Leguminosae, Papilionoideae), una Especie Nueva de la Depresión del Río Balsas, México." Novon a journal of botanical nomenclature from the Missouri Botanical Garden 19, 360-363.

View This Item Online: https://www.biodiversitylibrary.org/item/124658

Permalink: https://www.biodiversitylibrary.org/partpdf/121934

\section{Holding Institution}

Missouri Botanical Garden, Peter H. Raven Library

\section{Sponsored by}

Missouri Botanical Garden

\section{Copyright \& Reuse}

Copyright Status: Permission to digitize granted by rights holder Rights: https://www.biodiversitylibrary.org/permissions

This document was created from content at the Biodiversity Heritage Library, the world's largest open access digital library for biodiversity literature and archives. Visit BHL at https://www.biodiversitylibrary.org. 